\title{
The Relationship between Human Papillomavirus Infection and Cervical Precancerous Lesions
}

L. YU, X. MA, Y. HUA', X. YU1, N. YAN AND Z. LI' ${ }^{1 *}$

Department of Dermatology, The Affiliated Obstetrics and Gynecology Hospital of Nanjing Medical University, Nanjing Maternity and Child Health Care Hospital, 123 Tianfei Road, Nanjing,210004 Jiangsu, 'Department of Dermatology, The Second Hospital of Nanjing, Nanjing University of Chinese Medicine,1-1 Zhongfu Road, Nanjing, 210003 Jiangsu, China

Yu et al.: Human Papillomavirus Infection and Cervical Precancerous Lesions 
In order to explore the relationship between human papillary virus infection and cervical cancer, 1021 women were randomly selected from January 1, 2010 to December 31, 2018 in The Second Hospital of Nanjing. Thin-layer cytology test examination, colposcopy and cervical specimen analysis were carried out on all cases. Relevant data were collected and analyzed statistically. The results showed that in the analysis of single infection and multiple infection rates of human papillomavirus positive women, it was found that the multiple infection rate of human papillomavirus in women with high cervical precancerous lesions and cervical cancer was significantly higher than that in women with normal and inflammatory lesions and women with low cervical precancerous lesions $(p<0.01)$. In the analysis of the influence of age on the status of cervical cancer, it was found that the incidence of cervical high lesion increased significantly $(p<0.01)$ with increasing age. In the analysis of the integration status of human papillomavirus $\mathbf{1 6}$ genome, it was found that the integration degree of human papillomavirus 16 in cervical tissue cells was significantly $(p<0.01)$ correlated with the degree of cervical lesions. Therefore, this investigation revealed that the degree of cervical lesions could be controlled by interfering with the integration of human papillomavirus 16 in cervical tissue cells. Although there have been a few short comings, these results would still provide evidence and guidance for the treatment of cervical precancerous lesions by human papillomavirus.

\section{Key words: Cervical cancer, HPV, TCT, PCR reverse dot hybridization}

With the progress of economy peoples living standards are constantly improving. At the same time, human health has become the focus of attention. As one of the malignant tumors threatening women's life and health, the incidence of cervical cancer is also rising ${ }^{[1]}$. Currently, cervical cancer is gradually showing a younger trend ${ }^{[2]}$. At present, cervical cancer is the only cancer that is expected to be eliminated, because the etiology of cervical cancer has been clear, the human papillomavirus (HPV) infection is a high-risk factor among the risk factors of cervical cancer. HPV can be detected in all cervical cancer tissue samples. At the same time, it has been proved that persistent infection of high-risk HPV is very important in the occurrence of advanced cervical precancerous lesions ${ }^{[3,4]}$.

HPV is an epitheliophilic virus widely distributed in humans and other vertebrates with strict host and tissue specificity ${ }^{[5,6]}$. In recent years, many researchers have studied the correlation between these. HPV is the general name of a group of viruses, whose morphology is similar, but DNA restriction endonuclease maps are different and the antigenicity of nuclear shell proteins is different ${ }^{[7]}$. HPV is a group of deoxyribonucleic acid (DNA) viruses in subgroup A of the family Lactovaceae. It is highly species-specific and epitheliophilic. More than 140 subtypes of HPV have been found and about 40 subtypes can infect the reproductive system. According to the different risk of HPV in inducing malignant tumors of the reproductive tract, HPV can be divided into 2 groups, the low-risk and the high-risk. The highrisk HPV (HR-HPV) subtypes include HPV16, 18, 31,
$33,35,39,45,51,52,56,58,59$ and 68 , which often cause high-grade cervical intraepithelial neoplasia and other malignant lesions ${ }^{[8,9]}$. The cervical intraepithelial neoplasia caused by HR-HPV infection is a long-term and reversible precancerous lesion. Early intervention of cervical intraepithelial neoplasia can effectively prevent cervical cancer ${ }^{[10]}$. Therefore, not only in the prevention of cervical diseases, but also in guiding the clinical treatment of cervical diseases, evaluating the prognosis of cervical diseases, tracing the possibility of recurrence after treatment of cervical diseases, HPV detection has become an effective means and plays an important role ${ }^{[11]}$. Therefore, the research and prevention of this malignant tumor is a very necessary subject.

Although there are many studies on the effect of HPV on cervical cancer, there are few studies on the effect of HPV intervention on the treatment of cervical cancer. Therefore, 1021 women patients in the Affiliated Obstetrics and Gynecology Hospital of Nanjing Medical University were randomly selected as the research subjects to investigate the correlation between HPV and cervical cancer through collecting relevant data in order to provide new ideas for the treatment of cervical cancer in future.

About 1021 women were randomly selected from January 1, 2010 to December 31, 2018 in the Second Hospital of Nanjing. The age ranged from 17 to $59 \mathrm{y}$, with an average age of $37.3 \pm 5.2 \mathrm{y}$. All cases were examined by HPV-DNA + liquid-based thin-layer cytology test (TCT). If the test result is HPV $(+)$, the 
genotype and load of HPV needed to be analyzed. If HPV16 (+) is detected, its integration status needed to be analyzed. If there are abnormalities, cervical lesions should be diagnosed strictly according to the 3-step screening scheme for cervical disease diagnosis. The informed consent signed by the patients or their family members was obtained and this study was approved by the medical ethics committee of The Second Hospital of Nanjing.

All patients met with the following criteria, patients with an initial history of sexual life of $3 \mathrm{y}$ or more, no gynecological examination, vaginal medicine and cervical treatment in the past week, no previous history of other tumors, immunosuppressive diseases, use of immunosuppressive drugs, hysterectomy or cervical surgery, radiotherapy or chemotherapy and no pregnancy history for the near future. The patients have been informed and agreed to take relevant examinations without losing the interview. Exclusion criteria were pregnant women and patients with cervical cancer and precancerous lesions in the past. Patients who have undergone cervical loop electrosurgical excision procedure, conization and hysterectomy were excluded. Patients who have a history of pelvic radiotherapy or those on steroid hormone therapy were excluded.

Sterile cotton swab paper was used to gently wipe the excessive secretions of cervical mouth. The special HPV collector was inserted into the cervical canal, rotated clockwise for three cycles for $10 \mathrm{~s}$, slowly removed and placed in the special sampling tube marked with the code of the subject. The cap was tightened and the test tube was placed in a refrigerator at $-20^{\circ}$ for HR-HPV detection.

HR-HPV is detected using the HC-II method of Digene Company, USA. A total of 13 HR-HPV genotypes (HPV16, 18, 31, 33, 35, 39, 45, 51, 52, 56, 58, 59 and $68)$ were detected. The light generated by the sample was measured by DML2000 microplate reader and expressed as relative light unit (RLU). Positive control (PC) was established, which contained 1 pg/1 HPVDNA and about 5000 copies of HPV gene. The results were analysed using the ratio of RLU to standard positive control (RLU/PC). When RLU/PC $<1.0$, HPVDNA is negative, that is, HR-HPV infection is negative. When RLU/PC $>1.0$, HR-HPV infection is positive. Since the RLU value is proportional to the DNA contained in the sample, the higher the ratio is, the higher the HPVDNA load in the sample. Therefore, the RLU/PC value of the sample provided a semi-quantitative HPV load value. The RLU/PC values of HPV-DNA positive patients were divided into low load (1-10), moderate load (11-100) and high load (>100).

Reverse dot hybridization refers to the amplification of target nucleic acid by biotin-labeled primers and the hybridization of products with probes fixed on nylon membrane. A single hybridization reaction can detect multiple target sequences. DNA reverse dot blot hybridization combined with professional digital gel imaging and analysis system was used to detect HPV genotypes by $\mathrm{P}$ value (integral optical density difference). The cervical exfoliated cells sampler was inserted into the cervical canal to brush the cells and placed in a special specimen preservation solution. The HPV genotyping kit (Zhongshan Daan Gene Company), which can detect 15 HR-HPV genotypes (16, 18, 31, $33,35,39,45,51,52,53,56,58,59,66$, and 68) and four low-risk HPV genotypes $(6,11,43$, and CP8304) was used. People infected with two or more types of HPV are defined as multiple HPV infections.

According to the results of the typing test, the upstream and downstream primers of E2 and E6 were added to the system respectively. First, the total DNA was boiled for 7 min in boiling water at $100^{\circ}$ and then cooled rapidly. Then the PCR amplification reagent was added to the reaction system. Then $10 \mu 1$ of the PCR amplification product was sampled on $1.5 \%$ agarose gel and the control voltage was set at $60-80 \mathrm{~V}$. The electrophoresis was carried out for $50 \mathrm{~min}$ and UV Gel imaging system was used with Scion lmage4.0 electrophoretic image analysis software to measure and analyze the gray value of the electrophoretic strip area of the amplified products. The ratio of E2 to E6 was calculated and a set of data was obtained. The $95 \%$ reference value range of the data was analyzed. The lower limit was 0.80 . When the samples were measured 3 times, the mean value of E2/E6 was taken as the number of copies. When the E2/ E6 ratio was greater than or equal to 0.80 , HPV16 virus existed in a free state. When the E2/E6 ratio was less than 0.80 , the free and integrated HPV16 virus existed and when the E2/E6 ratio is 0, the HPV16 virus fully integrated.

Thin-layer cytology of cervix was examined on an automatic liquid-based thin-layer cytology machine (Becton Dickenson, USA). Thin-layer cytology smears with a diameter of $13 \mathrm{~mm}$ were made by sedimentation method. After fixed with $95 \%$ alcohol, Papanicolaou staining was performed. Cell morphology was observed under a microscope. Cytological diagnosis was made according to 2001 TBS system. The diagnosis results include no intraepithelial lesions or 
malignant lesions (NILM). The definite significance was atypical squamous cell (ASC-US), low-grade squamous intraepithelial lesion (LSIL), high-grade squamous intraepithelial lesion (HSIL) and squamous cell carcinoma (SCC). Cytological diagnosis results $\geq$ ASC-US was positive.

SLC-2000 electronic colposcope (Jinkuwei, Shenzhen) was used to observe cervical color, blood vessel and white spot with magnification of 20-40 times. Cotton balls dipped in $5 \%$ acetic acid solution were applied on the cervical surface, waiting for about $1 \mathrm{~min}$ to observe the boundary, color and blood vessel changes of lesions dynamically. The surface of cervix was coated with cotton ball dipped in $2 \%$ iodine solution to observe the distribution and situation of different iodine staining areas of cervix.

Colposcopy was performed for HPV positive patients and/or ASC-US positive patients. Cervical biopsy was performed for those with abnormal findings under colposcopy. The specimens were sent for pathological examination. If cytology is positive and no abnormal lesion or image is unsatisfactory under colposcopy, cervical biopsy or curettage at 3:00, 6:00, 9:00 and 12:00 were performed. The operation was performed by a specialist gynecologist and colposcopist. With pathological diagnosis as the gold standard, the pathological examination of cervical biopsy specimens was completed by 2 senior pathologists in the pathology department of The Second Hospital of Nanjing. SPSS22.0 statistical software was used and the measurement data were expressed as mean \pm standard deviation. One-way ANOVA is used to compare data among groups.
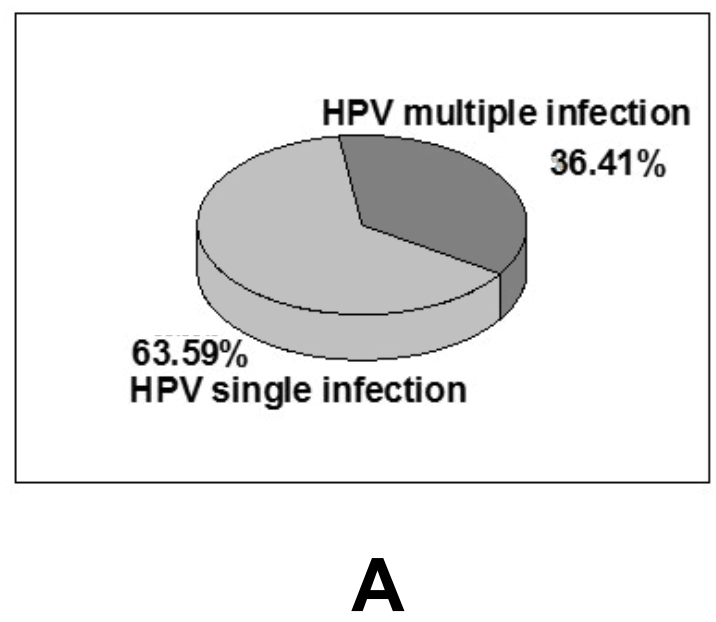

Fig. 1: Analysis of single and multiple infection rates in HPV-positive women

A. Pie chart of single and multiple infection rates of HPV in women with HPV positive genital tract; B. column chart of comparative analysis of multiple infection rates of HPV in each group, compared with normal and inflammation group, \#p $<0.01$
As shown in fig. 1, data were collected from 1021 patients in this study. After statistical analysis, it was found that there were 356 women with HPV positive genital tract. The single infection rate and multiple infection rate of HPV were 63.59 and $36.41 \%$, respectively. The multiple infection rate of HPV were $64.52 \%(40)$ and $61.90 \%(13)$ in women with high cervical precancerous lesions (62) and cervical cancer (21), respectively. Compared to the HPV multiple infection rate of 938 women in the inflammation group $(8.42 \%, 79$ cases) and 47 women in low-grade cervical precancerous lesions group $(17.02 \%, 8$ cases $)$, there was statistical significance $(\mathrm{p}<0.01)$.

As shown in fig. 2, the infection situation of patients with cervical lesions of different degrees in this study was analyzed. It was found that the rate of HPV infection in genital tract significantly $(\mathrm{p}<0.01)$ increased with the degree of cervical lesions. As can be seen from Table 1, the data collected in this investigation indicated significantly $(\mathrm{p}<0.01)$ with increasing age.

Fig. 3 shows that with the aggravation of cervical lesions, the incidence of viral integration in cervical tissue cells of HPV16 (+) patients gradually increased, while the incidence of viral dissociation gradually decreased. The proportion of HPV16 mixed infection in cervical tissue cells of cervical intraepithelial neoplasia (CIN) I and CIN III patients was the highest. In addition, the incidence of integration and mixing of HPV16 virus in cervical tissue cells increased with increasing cervical lesion grade. Statistical tests showed that the integration degree of HPV16 virus in cervical

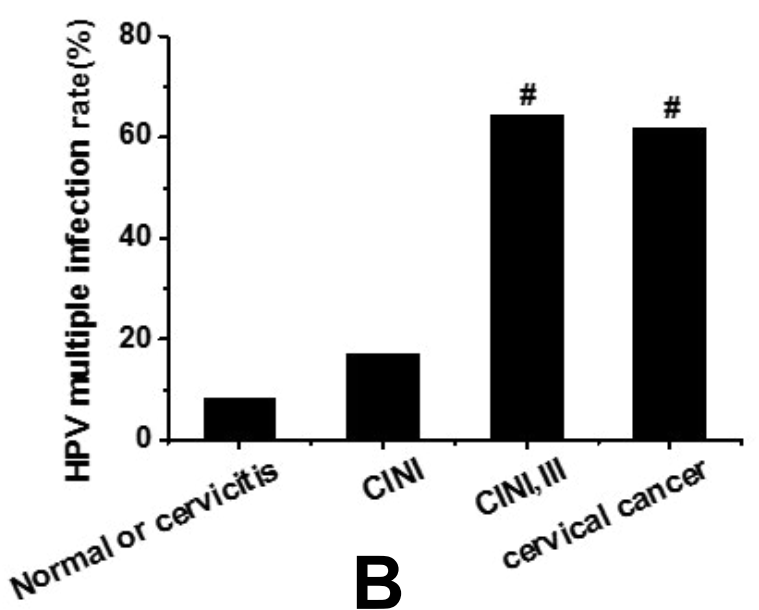
that the incidence of high cervical lesions increased 
tissue cells was significantly correlated with the degree of cervical lesions $(\mathrm{p}<0.01)$.

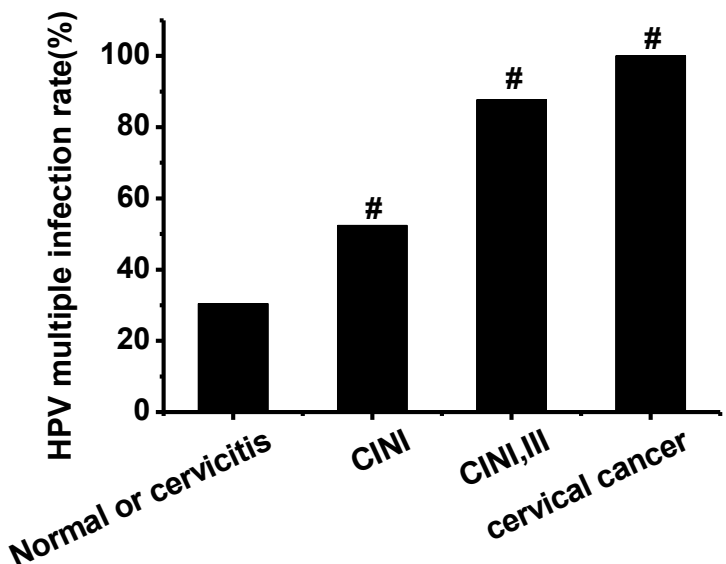

Fig. 2: Infection analysis in patients with cervical lesions of different degrees

Compared with normal and inflammation group, $\# p<0.01$

TABLE 1: ANALYSIS OF HPV INFECTION IN WOMEN OF DIFFERENT AGES

\begin{tabular}{lccc}
\hline Age & Pathology $\leq$ CIN I & Pathology $\geq$ CIN I & Total \\
\hline$\leq 30$ & $219(99.55 \%)$ & $1(0.45 \%)$ & 220 \\
$30-45$ & $311(95.99 \%)$ & $13(4.01 \%)$ & 324 \\
$>45$ & $452(94.76 \%)$ & $25(5.24 \%)$ & 477 \\
Total & 982 & 39 & 1021 \\
\hline
\end{tabular}
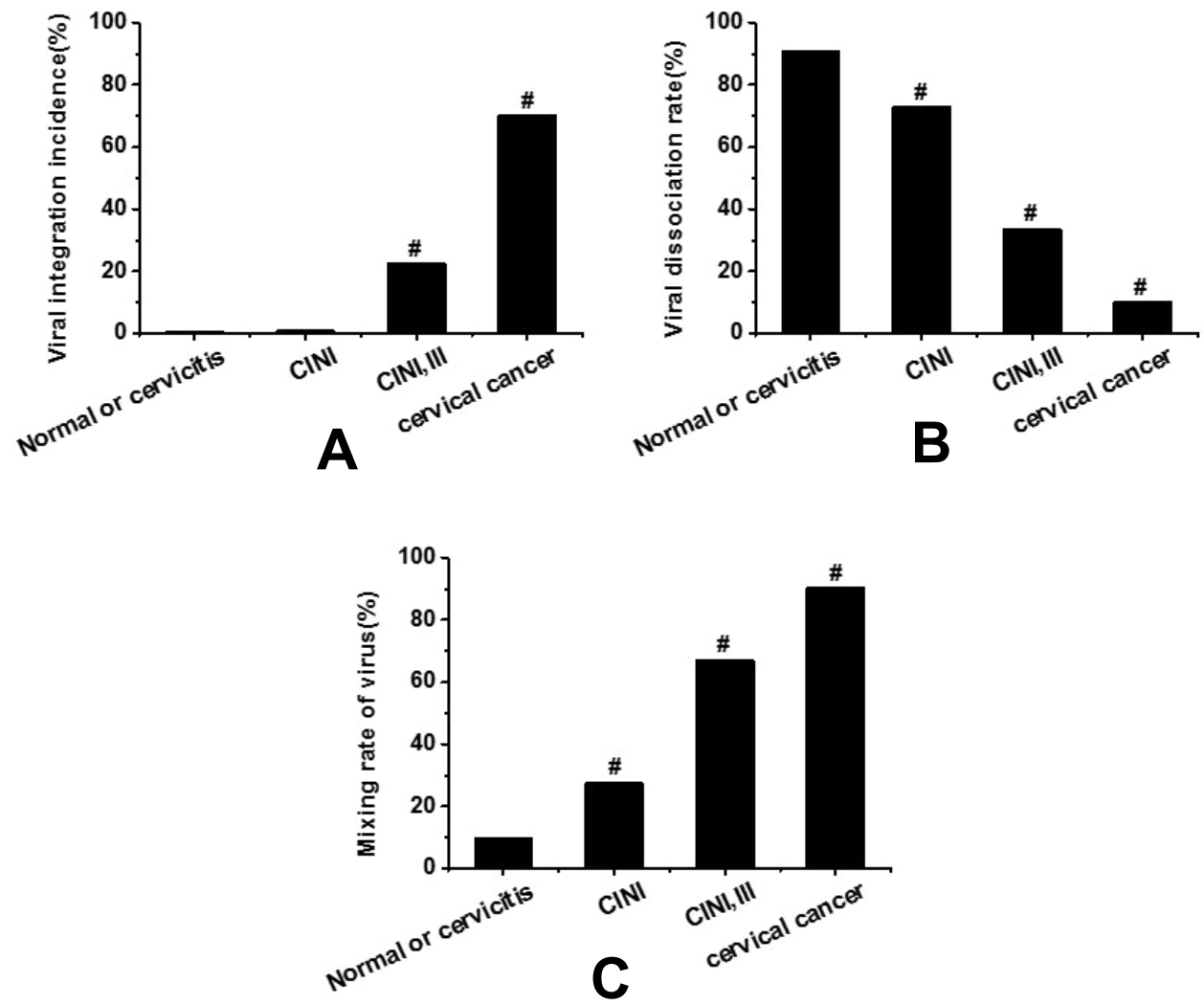

Fig. 3: HPV16 genome status map of cervical lesions of different degrees

A. the relationship with the incidence of viral integration; B. the relationship with the incidence of viral free; $C$. the relationship with the incidence of viral mixing, compared with normal and inflammation group, \#p $<0.01$ 
In conclusion, by exploring the relationship between HPV infection and cervical precancerous lesions, it was found that the degree of cervical lesions could be controlled by interfering with the integration of HPV16 virus in cervical tissue cells and the results were as expected. It provided experimental evidence for HPV treatment of cervical precancerous lesions. However, some shortcomings in this study such as smaller sample size and data, did not allow to make prediction on the mechanisms involved and it is necessary to increase data size to further explore mechanisms involved.

\section{REFERENCES}

1. Wang HR, Lin Y, Zhang XY, Ma XT. Transvaginal color doppler sonography combined with colposcopy for diagnosis of early stage cervical cancer and precancerous lesions. J Biol Regul Homeost Agents, 2018;32(1):123-126.

2. Lagheden C, Eklund C, Lamin H. Nationwide comprehensive human papillomavirus (HPV) genotyping of invasive cervical cancer. $\quad$ Br J Cancer 2018;118(10):1377.

3. Brisson M, Drolet M. Global elimination of cervical cancer as a public health problem. Lancet Oncol 2019;20(3):319-321.

4. de Martel C, Plummer M, Vignat J. Worldwide burden of cancer attributable to HPV by site, country and HPV type. Int J Cancer 2017;141(4):664-670.

5. Ottria L, Candotto V, Cura F. HPV acting on E-cadherin, p53 and p16: literature review. J Biol Regul Homeost Agents, 2018;32(2 Suppl. 1):73-79.

6. Miao H, Cao B, Ge W, Zhao W. Expression of p16 and p27 protein in cervical exfoliated cells and its relationship with high risk human papilloma virus in cervical lesions. J Biol Regul Homeost Agents 2019;33(1):197-203.

7. Gibson EG, Gage JC, Castle PE. Perceived Susceptibility to Cervical Cancer among African American Women in the Mississippi Delta: Does Adherence to Screening Matter. Women Health 2019;29(1):38-47.

8. Heller M, Cottrell C, Fish B. Cervical Cancer Screening in Underserved Populations: Reflex versus Co-testing for Cervical Cancer [16m]. Obstet Gynecol 2018;131:145S.

9. Modibbo F, Iregbu KC, Okuma J. Randomized trial evaluating self-sampling for HPV DNA based tests for cervical cancer screening in Nigeria. Infect Agents Cancer 2017;12(1):11.

10. Vaccarella S, Laversanne M, Ferlay J. Cervical cancer in Africa, Latin America and the Caribbean and A sia: Regional inequalities and changing trends. Int $\mathrm{J}$ Cancer 2017;141(10):1997-2001.

11. Chatterjee K, Mukherjee S, Vanmanen J. Dietary polyphenols, resveratrol and pterostilbene exhibit antitumor activity on an HPV E6-positive cervical cancer model An in vitro and in vivo analysis. Front Oncol 2019;9:352.

This is an open access article distributed under the terms of the Creative Commons Attribution-NonCommercial-ShareAlike 3.0 License, which allows others to remix, tweak, and build upon the work non-commercially, as long as the author is credited and the new creations are licensed under the identical terms

This article was originally published in a special issue: Special issue on "Drug Development and Human Health in China"

Indian J Pharm Sci 2020:82(1)spl issue2;105-110 